\section{What is outcomes- based CPD and what do I need to do?}

\section{Sue Paterson}

The Royal College of Veterinary Surgeons (RCVS) is introducing a new outcomes-based model of CPD. The new RCVS CPD model will focus on quality, impact and relevance of CPD rather than just counting hours. There has been increasing recognition over a range of different professions that CPD records based on 'inputs' alone, for example, measuring the number of hours attending a lecture, do not necessarily prove that any significant learning has taken place or that this learning will be used to improve professional practice. By contrast, research has demonstrated that CPD activities focused on outcomes encourage professionals to reflect on what they have learned, how they will apply their learning and how it will improve their practice, which has a positive impact on professionalism and patient health outcomes.

In this session the chair of RCVS Education Committee, Sue Paterson, will describe the changes to the CPD requirement, the benefits of an outcome-based model and demonstrate how easy it will be to record and reflect your CPD using the new 1CPD app.

\section{KEY LEARNING OBJECTIVES}

Be able to outline the benefits of an outcome-based continuing professional development (CPD) model Understand the stages of the CPD cycle Know how to record CPD and reflections using the new CPD recording platform and app

\section{MULTIPLE CHOICE QUESTIONS}

1. What is the focus of an outcomes-based CPD model?
(A) The impact the CPD that has had on your behaviour or in your role
(B) Counting hours
(C) External CPD events
(D) To develop new knowledge outside of your role

2. What is the name of the RCVS' new recording portal?
(A) RCVS CPD
(C) CPDaRCVS
(B) 1CPD
(D) My learning portal

3. What can be counted as CPD according to the RCVS' CPD Policy?

(A) Only clinical CPD

(B) Formal or face-to-face learning

(C) Any learning or development that is relevant to you as a professional

(D) Courses, congresses and online learning

\section{Mind Matters initiative: about Schwartz Rounds; experience a Schwartz Round}

\section{Amy Martin}

Part one of this double session will give you an overview of the Schwartz Rounds model, which is being piloted by the Point of Care Foundation and the RCVS-led Mind Matters initiative in a selection of UK veterinary practices.

A representative of the Point of Care Foundation, the charity that delivers Schwartz Round training and support in the UK, will discuss how this group reflective approach can provide an opportunity for staff from all roles within the veterinary team to come to terms with the emotional aspects of work. It is a model that has brought excellent results in the human medical field and, via this pilot, it is hoped that these benefits can be transferred into the veterinary sector.

The talk will then be followed by an opportunity to experience a Schwartz Round live, led by Amy Martin,
Practice Manager from Holly House Vets, the first practice in the UK to adopt Schwartz Rounds. This stimulating interactive session will give you an understanding of the approach and its impact.

Both sessions will be suitable for any member of the veterinary team.

\section{KEY LEARNING OBJECTIVES}

- Understand the impact of reflection in terms managing the emotional impact of veterinary work

- Understand how a Schwartz Round works

- Experience at first-hand how the model might work for your practice

\section{MULTIPLE CHOICE QUESTIONS}

1. Who is able to attend a Schwartz Round?

(A) Only members of the clinical team

(B) Only front of house staff

(C) Only management

(D) All members of the team 